\title{
ON THE STOKES FLOW COMPUTATION ALGORITHM BASED ON WOODBURY FORMULA
}

\author{
A. I. Popov ${ }^{1}$, I. S. Lobanov ${ }^{1}$, I. Yu. Popov ${ }^{1}$, T. V. Gerya ${ }^{2}$ \\ ${ }^{1}$ ITMO University, Kronverkskiy 49, 197101, St. Petersburg, Russia \\ ${ }^{2}$ Institute of Geophysics, Department of Earth Sciences, Swiss Federal Institute \\ of Technology Zurich (ETH), 5 Sonnegstrasse, CH-8092 Zurich, Switzerland \\ popov239@gmail.com, taras.gerya@erdw.ethz.ch
}

PACS 47.10.ad, 47.11.Fg

DOI 10.17586/2220-8054-2015-6-1-140-145

The Stokes approximation is used for the description of flow in nanostructures. An algorithm for Stokes flow computation in cases when there is great variation in the viscosity over a small spatial region is described. This method allows us to overcome computational difficulties of the finite-difference method. The background of the approach is using the Woodbury formula - a discrete analog of the Krein resolvent formula. The particular example of a rectangular domain is considered in detail. The inversion of the discrete Stokes operator is made in analytic form for the case of constant viscosity.

Keywords: nanotube, Stokes flow, finite-difference method.

Received: 1 December 2014

Revised: 4 January 2015

\section{Introduction}

Flow in nanostructures have many peculiarities which are not explained by the conventional theory of fluid flow [1,2]. At present, there is no general theory of nanoflows. We can only mention a few specific models [3-5]. Experiments show that nano-sized confinement leads to strong variation of liquid viscosity within the nanostructure [6-8]. One observes a similar situation when dealing with flow with nanoparticles or with singularities (see, e.g., $[9,10]$ ). Additionally, there is a fraction separation effect [11]. This is an indirect confirmation of the crystalline model of nanoflow which is based on assumption that nanoliquid contains solid-like inclusions. To describe such flow in the framework of the model it is necessary to solve the Stokes equation with strong variation in viscosity. This creates difficulties for computations, particularly for the finite difference scheme [12-15]. To overcome these obstacles an approach based on the Woodbury formula was suggested [16]. The formula reduces the inversion of the discrete Stokes operator with small inclusions having strong viscosity contrast to the inversion of the operator with constant (or slowly varying) viscosity and the inversion of small rank operator. In the present paper, we consider in detail the first step.

In more detail, the governing equations in $d$-dimensional $(\mathrm{d}=2,3)$ case (i.e. the Stokes and continuity equations for velocity $v$ and pressure $p$ under the action of force $F$ ) can be written as follows:

$$
\frac{\partial}{\partial x_{j}}\left(\eta\left(\frac{\partial v_{i}}{\partial x_{j}}+\frac{\partial v_{j}}{\partial x_{i}}\right)\right)-\frac{\partial p}{\partial x_{i}}=F_{i}, \frac{\partial v_{i}}{\partial x_{i}}=0
$$


We assume that the viscosity $\eta$ has strong variation in small spatial domain and is constant outside of it. We use finite-difference discretization on non-staggered grid:

$$
\left\{\begin{array}{l}
h_{j}^{-1} \Delta_{j}\left(\eta\left(h_{j}^{-1} \nabla_{j} v_{i}+h_{i}^{-1} \nabla_{i} v_{j}\right)\right)-h_{i}^{-1} \nabla_{i} p=F_{i}, \\
h_{i}^{-1} \Delta_{i} v_{i}=0
\end{array}\right.
$$

where $h_{i}$ is a mesh size, $\Delta_{i}$ is a forward difference, $\nabla_{i}$ is a backward difference,

$$
\begin{aligned}
& \Delta_{j} f\left(x_{1}, \ldots, x_{d}\right)=f\left(x_{1}, \ldots, x_{i}+h_{i}, \ldots, x_{d}\right)-f\left(x_{1}, \ldots, x_{i}, \ldots, x_{d}\right), \\
& \nabla_{j} f\left(x_{1}, \ldots, x_{d}\right)=f\left(x_{1}, \ldots, x_{i}, \ldots, x_{d}\right)-f\left(x_{1}, \ldots, x_{i}-h_{i}, \ldots, x_{d}\right) .
\end{aligned}
$$

The Woodbury formula allows us to improve the procedure of solving equations (2) in the case of strong viscosity variation.

\section{Woodbury formula}

Let us briefly describe the finite-difference approach based on the Woodbury formula (as for details, see [16]). Consider the discrete Stokes operator $S$ on the lattice:

$$
S V_{i}=\triangle_{j}\left(\eta\left(\nabla_{j} V_{i}+\nabla_{i} V_{j}\right)\right),
$$

where $V$ is the liquid velocity, $\eta$ is the viscosity. Symbols $\Delta_{p}$ and $\nabla_{p}$ are used, correspondingly, for the forward and the backward finite differences for coordinate $p$ where we assume for simplicity (it is not a limitation) that the lattice has a unit step. We use the following operator representation:

$$
S=S_{a}+S_{p}^{T}, \quad S_{a}:=\eta S_{0}
$$

where $S_{0}$ is the Stokes operator with the viscosity equals one:

$$
S_{0} V_{i}=\sum_{j}\left(\triangle_{j} \nabla_{j} V_{i}+\triangle_{j} \nabla_{i} V_{j}\right)
$$

and:

$$
S_{p}^{T} V_{i}=\left(\triangle_{j} \eta\right) T_{j}\left(\nabla_{j} V_{i}+\nabla_{i} V_{j}\right)
$$

Here $T_{i}$ is the translation operator:

$$
T_{i} f\left(x_{1}, \ldots, x_{d}\right)=f\left(x_{1}, \ldots, x_{i}+h_{i}, \ldots, x_{d}\right) .
$$

Note that the operator $S_{p}$ has small rank due to the property of the viscosity $\eta$, i.e. the corresponding matrix has many zero entries. We would like to solve the following equation:

$$
S V=G,
$$

where $G$ is preassigned vector. To find a solution in our case it is convenient to use the Woodbury formula:

$$
V_{i}=S^{-1} G_{i}=\left(\eta S_{0}+S_{p}\right)^{-1} G=\left[1-\left(\eta S_{0}\right)^{-1}\left(1+S_{p}^{T}\left(\eta S_{0}\right)^{-1}\right)^{-1} S_{p}^{T}\right]\left(\eta S_{0}\right)^{-1} G
$$

One can see that we have real computational improvement (due to small rank of $S_{p}^{T}$ ) if we can find the inverse operator $\left(\eta S_{0}\right)^{-1}$ without high computational complexity. It is really so. We can find the inverse operator for the case of constant viscosity, e.g., by using of the discrete Fourier transform. Namely, let:

$$
X=\left(\eta S_{0}\right)^{-1} G=S_{0}^{-1} \eta^{-1} G .
$$

To find $X$ one should solve the equation:

$$
\eta S_{0} X=G
$$


After the Fourier transformation we obtain:

$$
\hat{S}_{0} \hat{X}=\eta^{-\hat{1}} G \Leftrightarrow \hat{X}=\left(\hat{S}_{0}\right)^{-1} \eta^{-\hat{1}} G
$$

These operations are not associated with high complexity.

In the next section we consider in detail how to use the discrete Fourier transform in a particular case.

\section{Discrete Stokes operator inversion for the case of constant viscosity in a rectangle}

To find the inverse matrix we use the discrete Fourier transform. To introduce it we need the corresponding system of eigenfunctions. Let us choose the system.

Consider the discrete Stokes equations coupled with the discrete continuity equation for the case of constant viscosity $\eta=1$ :

$$
\left\{\begin{array}{l}
-2 \Delta_{1} \nabla_{1} V_{1}-\Delta_{2}\left(\nabla_{2} V_{1}+\nabla_{1} V_{2}\right)+\nabla_{1} P=F_{1}, \\
-2 \Delta_{2} \nabla_{2} V_{2}-\Delta_{1}\left(\nabla_{1} V_{2}+\nabla_{2} V_{1}\right)+\nabla_{2} P=F_{2}, \\
\Delta_{1} V_{1}+\Delta_{2} V_{2}=0 .
\end{array}\right.
$$

We deal with vector fields $V$ and $F$ and scalar field $P$, determined on a two-dimensional lattice with $N=L^{2}$ nodes. The nodes are numbered by vectors with integer terms $x=$ $\left(x_{1}, x_{2}\right), x_{k} \in \mathcal{D}=[0, L] \cap \mathbb{Z}$. Symbols $\Delta_{p}$ and $\nabla_{p}$ are used, correspondingly, for the forward and the backward finite differences for coordinate $p$ :

$$
\begin{array}{ll}
\Delta_{1} f(x)=f\left(x_{1}+1, x_{2}\right)-f(x), & \nabla_{1} f(x)=f(x)-f\left(x_{1}-1, x_{2}\right), \\
\Delta_{2} f(x)=f\left(x_{1}, x_{2}+1\right)-f(x), & \nabla_{2} f(x)=f(x)-f\left(x_{1}, x_{2}-1\right),
\end{array}
$$

where we assume for simplicity (it is not a limitation) that the lattice has a unit step. We will solve the system (3) with the free slip boundary condition:

$$
\left\{\begin{array}{l}
\left.V_{k}\right|_{x_{k}= \pm L}=0 \\
\left.\nabla_{j} V_{k}\right|_{x_{j}= \pm L}=0, \quad j \neq k .
\end{array}\right.
$$

Consider a one-dimensional lattice $\mathcal{D}$ and the family of functions $\phi_{n}, n=1 . . L-1$ determined on the lattice by the following expressions:

$$
\phi_{n}(t)=\sin \frac{\pi n t}{L}, \quad t \in \mathcal{D} .
$$

One can verify that the functions $\phi_{n}$ form a basis in the space of functions on the lattice $\mathcal{D}$, satisfying the conditions:

Consider the operator:

$$
\phi_{j}(0)=\phi_{j}(L)=0
$$

$$
\Delta \nabla f(t)=\nabla f(t+1)-\nabla f(t)=f(t+1)-2 f(t)+f(t-1) .
$$

It is simple to verify that $\Delta \nabla=\nabla \Delta)$. Direct calculation gives us:

$$
\Delta \nabla \phi_{j}(t)=\Delta \nabla \sin \frac{\pi j t}{L}=\sin \frac{\pi j(t+1)}{L}-2 \sin \frac{\pi j t}{L}+\sin \frac{\pi j(t-1)}{L}=2 \sin \frac{\pi j t}{L}\left(\cos \frac{\pi j}{L}-1\right),
$$

hence,

$$
\Delta \nabla \phi_{j}=\nabla \Delta \phi_{j}=\alpha_{j} \phi_{j}, \quad \alpha_{j}=2\left(\cos \frac{\pi j}{L}-1\right)
$$

We introduce the notation:

$$
\theta_{j}=\Delta \phi_{j} .
$$


Then,

$$
\nabla \theta_{j}=\nabla \Delta \phi_{j}=\alpha_{j} \phi_{j}
$$

Particularly,

$$
\nabla \theta_{j}(d)=\alpha_{j} \phi_{j}(d)=0, \quad \nabla \theta_{j}(0)=0
$$

One can verify that $\theta_{j}$ is also the eigenvector of the operator of second symmetric finite difference corresponding to the same eigenvalue as $\phi_{j}$. Actually,

$$
\Delta \nabla \theta_{j}=\Delta \nabla \Delta \phi_{j}=\Delta \alpha_{j} \phi_{j}=\alpha_{j} \theta_{j} .
$$

We additionally determine $\theta_{0} \equiv 1$ and $\phi_{0} \equiv 0$, which satisfy all the above-described properties.

Consider the family of functions $\Psi_{j}$, where the vector index $j=\left(j_{1}, j_{2}\right)$ has coordinates $j_{p}=0 . . L-1$ :

$$
\Psi^{j}(x)=\theta_{j_{1}}\left(x_{1}\right) \theta_{j_{2}}\left(x_{2}\right) .
$$

Let us seek the solution of equation (3) in the form:

$$
V_{k}(x)=\sum_{j} V_{k}^{j} \nabla_{k} \Psi^{j}(x), k=1,2, \quad P(x)=\sum_{j} P^{j} \Psi^{j}(x),
$$

for some real coefficients $V_{k}^{j}, P^{j}$. One can see that the expression of such type satisfies the boundary conditions (4). One can show that any vector field $V$ satisfying the conditions (4) can be represented in the form of (11). But arbitrary pressure field $P$, generally speaking, can not be presented in such form. Now we consider the component related to the velocity field only.

Substituting (11) into the Stokes equation from (3), one obtains:

$$
-\sum_{p=1}^{2} \Delta_{p}\left(\nabla_{p} \sum_{j} V_{k}^{j} \nabla_{k} \Psi^{j}+\nabla_{k} \sum_{j} V_{p}^{j} \nabla_{p} \Psi^{j}\right)+\nabla_{k} \sum_{j} P^{j} \Psi^{j}=\sum_{j} F_{k}^{j} \nabla_{k} \Psi^{j}, \quad k=1,2,
$$

where $F_{k}^{j}$ is the projection of $F_{k}$ on $\Psi^{j}$. Changing the order of summation, one gets:

$$
\sum_{j}\left[P^{j} \nabla_{k} \Psi^{j}-F_{k}^{j} \nabla_{k} \Psi^{j}-\sum_{p=1}^{N}\left(V_{k}^{j} \Delta_{p} \nabla_{p} \nabla_{k} \Psi^{j}-V_{p}^{j} \Delta_{p} \nabla_{k} \nabla_{p} \Psi^{j}\right)\right]=0 .
$$

Using the commutativity $\Delta_{n} \nabla_{m}=\nabla_{m} \Delta_{n}$ and the property $\Delta_{p} \nabla_{p} \Psi^{j}=\alpha_{j_{p}} \Psi^{j}$, One finds that

$$
\sum_{j}\left[P^{j}-F_{k}^{j}-\sum_{p=1}^{2} \alpha_{j_{p}}\left(V_{k}^{j}-V_{p}^{j}\right)\right] \nabla_{k} \Psi^{j}=0 .
$$

Due to linear independence of the vectors $\nabla_{k} \Psi^{j}$, the Stokes equation is equivalent to a set of systems:

$$
P^{j}-\sum_{p=1}^{2} \alpha_{j_{p}}\left(V_{k}^{j}-V_{p}^{j}\right)=F_{k}^{j}, \quad k=1,2, \forall j .
$$

Substituting (11) into the continuity equation from (3), we come to the equation:

$$
\sum_{p=1}^{2} \Delta_{p} \sum_{j} V_{p}^{j} \nabla_{p} \Psi^{j}(x)=0
$$


After simplification one gets:

$$
\sum_{j} \sum_{p=1}^{2} \alpha_{j_{p}} V_{p}^{j} \Psi^{j}(x)=0
$$

Due to linear independence of $\Psi^{j}$, the system is equivalent to the following one:

$$
\sum_{p=1}^{2} \alpha_{j_{p}} V_{p}^{j}=0 \quad \forall j
$$

Finally, the problem (3) with the boundary conditions (4) is reduced to the set of systems:

$$
\left\{\begin{array}{l}
P^{j}-\sum_{p=1}^{2} \alpha_{j_{p}}\left(V_{k}^{j}-V_{p}^{j}\right)=F_{k}^{j} \quad k=1,2, \forall j \\
\sum_{p=1}^{2} \alpha_{j_{p}} V_{p}^{j}=0 \quad \forall j .
\end{array}\right.
$$

Using the second equation, one can simplify the first one:

$$
\left\{\begin{array}{l}
P^{j}-V_{k}^{j} \sum_{p=1}^{2} \alpha_{j_{p}}=F_{k}^{j} \quad k=1,2, \forall j \\
\sum_{p=1}^{2} \alpha_{j_{p}} V_{p}^{j}=0 \quad \forall j .
\end{array}\right.
$$

Multiplying the first equation by $\alpha_{j_{k}}$ and making a summation by $k$, one gets:

$$
P^{j}\left(\sum_{k} \alpha_{j_{k}}\right)-\left(\sum_{k} \alpha_{j_{k}} V_{k}^{j}\right)\left(\sum_{p=1}^{2} \alpha_{j_{p}}\right)=\left(\sum_{k} \alpha_{j_{k}} F_{k}^{j}\right) \quad \forall j,
$$

Due to the second equation of the system, one has:

$$
P^{j}=\frac{\sum_{p=1}^{2} \alpha_{j_{p}} F_{p}^{j}}{\sum_{p=1}^{2} \alpha_{j_{p}}} \forall j .
$$

Hence, the first equation gives us the velocity field:

$$
V_{k}^{j}=\frac{P^{j}-F_{k}^{j}}{\sum_{p=1}^{2} \alpha_{j_{p}}} \quad k=1,2, \forall j
$$

Thus, for this particular case we obtained the solution in an explicit form. As for more complicated domain, one can follow the suggested procedure if the system of eigenfunctions is known. In this case the approach described allows one to obtain essential acceleration and guaranteed convergence of the computations for strongly varying viscosity.

\section{Acknowledgements}

This work was partially financially supported by the Government of the Russian Federation (grant 074-U01), by the Ministry of Science and Education of the Russian Federation (GOSZADANIE 2014/190, Project 14.Z50.31.0031), by grant MK-5001.2015.1 of the President of the Russian Federation.

\section{References}

[1] Li D. (Ed.) Encyclopedia of microfluidics and nanofluidics. New York: Springer (2008).

[2] Rivera J. L., Starr F. W. Rapid transport of water via carbon nanotube syringe. J. Phys. Chem. C., 114, P. 3737--3742 (2010).

[3] Chiviulikhin S.A., Gusarov V.V., Popov I.Yu. Flows in nanostructures: hybrid classical-quantum models. Nanosystems: Phys. Chem. Math., 3(1), P. 7-26 (2012).

[4] Hanasaki I., Nakatani A. Fluidized piston model for molecular dynamics simulations of hydrodynamic flow. Modelling Simul. Mater. Sci. Eng., 14, P. 9-20 (2006). 
[5] Maslov V.P. Superfluidity of classical liquid in a nanotube for even and odd numbers of neutrons in a molecule. Theor. Math. Phys., 153, P. 1677--1796 (2007).

[6] Li T.-D., Gao J., Szoszkeiwicz R., Landman U., Riedo E. Structured and viscous water in subnanometer gaps. Phys. Rev. B., 75, P. 115415-1-115415-6 (2007).

[7] Mashl R. J., Joseph S., Aluru N. R., Jakobsson E. Anomalously Immobilized Water: A New Water Phase Induced by Confinement in Nanotubes. Nano Letters, 3, P. 589-592 (2003).

[8] Popov I.Yu. Statistical derivation of modified hydrodynamic equations for nanotube flows. Phys. Scripta, 83, P. 045601/1-3 (2011).

[9] Batischev V.A., Zaikin V.V., Horoshunova E.V. Heat transport in Marangoni layer with nanoparticles. Nanosystems: Physics, Chemistry, Mathematics, 4(3), P. 313-319 (2013).

[10] Blinova I.V. Model of non-axisymmetric flow in nanotube. Nanosystems: Physics, Chemistry, Mathematics, 4(3), P. 320-323 (2013).

[11] Kononova S. V. et. al. Polimer-inorganic nanocomposites based on aromatic polyamidoimides effective in the process of liquids separation. Russ. J. Gen. Chem., 80, P. 1136--1142 (2010).

[12] T. Gerya. Introduction to Numerical Geodynamic Modelling. Cambridge University Press, Cambridge (2009).

[13] A. Ismail-Zadeh, P.Tackley. Computational Methods for Geodynamics. Cambridge University Press, Cambridge (2010).

[14] T.Duretz, D. A. May, T. V. Gerya, P. J. Tackley, Discretization errors and free surface stabilization in the finite difference and marker-in-cell method for applied geodynamics: A numerical study. Geochem. Geophys. Geosyst., 12 (2011), Q07004, doi:10.1029/2011GC003567.

[15] Popov I.Y., Lobanov I.S., Popov A.I., Gerya T.V. Benchmark solutions for nanoflows. Nanosystems: Physics, Chemistry, Mathematics, 5(3), P. 391-399 (2014).

[16] Popov I.Y., Lobanov I.S., Popov A.I., Gerya T.V. Numerical approach to the Stokes problem with high contrasts in viscosity. Applied Mathematics and Computations, 235, P. 17-25 (2014). DOI 10.1016/j.amc.2014.02.084. 